\title{
The usefulness of fleet rectal enemas on high-dose-rate intracavitary cervical cancer brachytherapy. A prospective trial
}

\author{
Ignacio Andres, MD', Manuel Gutierrez-Perez, RN', Maria Pilar Rodriguez-Vela, RTT', Roberto Berenguer, MSc', \\ Marimar Sevillano, MD', Manuel Aguayo, MD', Meritxell Arenas, PhD², Angeles Rovirosa, PhD³, \\ Yashmina Murria-Perez, MD', Sebastia Sabater, MD, PhDl \\ 'Department of Radiation Oncology, Complejo Hospitalario Universitario de Albacete (CHUA), Albacete, ${ }^{2}$ Department of Radiation \\ Oncology, Hospital Universitari Sant Joan, Reus, ${ }^{3}$ Gynecological Cancer Unit, Radiation Oncology Department, ICMHO, Hospital Clinic, \\ University of Barcelona, IDIBAPS, Barcelona, Spain
}

\begin{abstract}
Purpose: To evaluate the effects of rectal enemas on rectal doses during radical high-dose-rate (HDR) intracavitary cervical brachytherapy (BT).

Material and methods: Twenty patients suffering from cervical cancer and treated with external beam radiotherapy and HDR-BT were included in a prospective trial. The first brachytherapy fraction was considered the basal status, and patients were instructed to self-administer two rectal cleansing enemas before the second fraction. Dose-volume histogram (DVH) values were generated for the rectum and correlated with rectal volume variation. Brachytherapy was carried out with a Fletcher or Utrecht applicator.

Results: No significant rectal volume differences were observed between fractions with or without rectal enemas (without, $52.64 \pm 15.92 \mathrm{cc}$; with, $53.16 \pm 19.28 \mathrm{cc}$ ). There was a significant correlation between both rectal volumes $(r=0.722, p=0.001)$. No significant differences were observed in analyzed DVH parameters (median values: $\Delta \mathrm{D}_{0.1 \mathrm{cc}}$ 4.17 vs. $3.61 \mathrm{~Gy} ; \Delta \mathrm{D}_{1 \mathrm{cc} c^{\prime}} 3.23$ vs. $2.87 \mathrm{~Gy} ; \Delta \mathrm{D}_{2 \mathrm{cc}} 2.9$ vs. $2.54 \mathrm{~Gy} ; \Delta \mathrm{D}_{5 \mathrm{cc} c^{\prime}} 2.35$ vs. $2.05 \mathrm{~Gy}$, for no enema and enema fraction, respectively). No significant rectal volume differences nor DVH parameter differences were observed according the applicator type.

Conclusions: Our rectal enemas protocol prior to HDR-BT was ineffective in significantly modifying rectal DVH parameters. No differences were observed according to the type of applicator used.

J Contemp Brachytherapy 2017; 9, 3: 224-229 DOI: https://doi.org/10.5114/jcb.2017.68135
\end{abstract}

Key words: cervical brachytherapy, cervical cancer, dose-volume histogram, rectal enema.

\section{Purpose}

Intracavitary cervical brachytherapy (BT) is a cornerstone in the treatment of cervical cancer combined with external beam radiotherapy (EBRT) because it delivers a high dose to the primary cervical tumor and adjacent soft tissues, resulting in increased local control and survival $[1,2,3,4]$. The rectum is the main organ at risk (OAR) for complications [5]. Classically, rectal and bladder points on orthogonal radiographs have represented the doses to these organs and have been used to estimate the risk of complications [6]. These points only provide a limited information of the OAR anatomy and its relationship with the brachytherapy applicator. The availability of tree-dimensional images (3D), computed tomography (CT), or magnetic resonance imaging (MRI) have revealed in- consistencies of the OARs doses between 3D and planar dose-calculation methods [7]. The availability of tridimensional images, computed tomography (CT), or magnetic resonance imaging (MRI) have shown that these points do not take the exact tumor and OAR anatomy into account [7]. Up to $23 \%$ of the patients can experience rectal toxicity grade 2 or higher [8]. Higher doses per fraction and higher doses at point $\mathrm{A}$ have been shown to increase rectal toxicity [6].

The long-lasting low-dose-rate (LDR) applications with several hospital rest days avoid rectal discharge for applications lasting longer than two days [9], and rectal enemas prior to the procedure have been advised [10]. Rectal evacuation was also recommended for LDR vaginal cuff brachytherapy (VCB) [9]. Our group was concerned about the relationship between rectal filling and
Address for correspondence: Sebastia Sabater, MD, PhD, Department of Radiation Oncology, Complejo Hospitalario Universitario de Albacete (CHUA), C/ Hnos Falcó 37, 02006. Albacete, Spain, phone: +34 967597523, fax: +34 967597530, e-mail: ssabaterm@gmail.com
Received: 08.11.2016 Accepted: 29.04.2017 Published: 30.06 .2017 
rectal doses, as well as the value of keeping rectal cleansing practices in the current gynecological high-dose-rate (HDR) brachytherapy setting $[11,12,13]$. We have recently published a trial analyzing the effect of rectal enemas on rectal dosimetric parameters during VCB [14], and here we report a parallel study on cervical cancer during definitive HDR-brachytherapy after EBRT.

\section{Material and methods}

Patients' eligibility criteria included a histologically confirmed diagnosis of cervical cancer, no evidence of distant metastases other than para-aortic lymph node metastases, no history of abdominal pelvic RT, an Eastern Cooperative Oncology Group performance status of 0-2, and no known history of inflammatory bowel disease. The patients' treatment followed standard protocol, apart from the inclusion of the rectal enema administered prior to the second fraction.

Patients were staged with a physical examination, blood analysis, and MRI of the pelvis and abdomen. Additional staging procedures were performed on a patient by patient basis. Radiotherapy consisted of EBRT, followed by intracavitary BT performed under general anesthetic. All patients received concomitant weekly cisplatin during EBRT. EBRT and BT courses were administered in the outpatient setting. Initially, the whole pelvis was treated with $6 / 18 \mathrm{VM}$ photons with a 3D conformal four-field box technique up to 44 to 50.4 Gy in 22 to 28 fractions. Parametriums were boosted to 60-66 Gy to patients with International Federation of Gynecology and Obstetrics (FIGO) stage IIb-III. Five HDR-BT fractions were administered twice a week using a ${ }^{192}$ Ir remote afterloading technique (MicroSelectron HDR, Elekta, Stockholm, Sweden) concurrently with parametrial boosting or the last EBRT fractions. The HDR-BT was performed using a Fletcher-style applicator. The first 10 patients were treated with a metallic Fletcher-Williamson applicator (part \#110.270A01, Nucletron, Elekta, Stockholm, Sweden) with unshielded mini-colpostats because the large number of artefacts on CT images caused by the shield material inside colpostats, preclude the use of larger colpostats. Afterwards, the metallic applicator was replaced by a CT/MR compatible Utrecht applicator and colpostats with a rectal retractor (part \#085.230, Nucletron, Elekta, Stockholm, Sweden). No interstitial needles were used. The standard prescribed dose was 5 Gy to point A. The length of the intrauterine tandem and distance between colpostat was adjusted to the patients' anatomy at each BT fraction. Anterior and posterior gauze packs were used to stabilize the applicator, and to push the bladder and the rectum away.

The first and second BT fractions were used for the study; the first being considered the basal or reference status, and the second being the experimental rectal cleansing. A pelvic CT scan was routinely carried out at every fraction, with a $2 \mathrm{~mm}$ slice thickness and no gap between slices, in the supine position. Patients were instructed to try to evacuate before coming to the hospital for HDR-BT, and two Fleet enemas were self-administered before the second fraction: the first the night before the procedure, and the second, at home, before the hospital admittance.
Computed tomography images were transferred to a 3D treatment planning system (Oncentra v. 4.1, Elekta, Stockholm, Sweden) in order to create a customized plan at every fraction. The entire outer bladder wall was segmented and the rectum was delineated from the rectosigmoid union to $1 \mathrm{~cm}$ below the femoral heads by the same physician. The tandem active length was individualized to the patients' anatomy at every fraction. Optimization was employed based on points. The points were placed around tandem and colpostats following the American Brachytherapy Society protocol [15], and using the volume option.

\section{Statistical analysis}

A sample size of 52 patients was calculated based on an estimated difference in mean rectum volume of $7 \pm 20 \mathrm{cc}$ between both CTs $(\beta=80 \%, \alpha=0.05$, two sided test). It was calculated taking into account previous results that demonstrated a rectal volume reduction of $22 \mathrm{cc}$ after rectal deflation in patients with rectal gas pockets [13]. The expected volume difference was thought to be two-thirds less $(7 \mathrm{cc})$ than our previous study. The Institutional Review Board approved this study, and before brachytherapy, informed consents were obtained from all study patients.

Results are shown as mean ( \pm standard deviation) or as median (interquartile range [iqr]) for non-normal data. Normality was assessed with the Kolmogorov-Smirnoff test. Bladder and rectal volume differences at the first (without Fleet enemas) and second BT fraction (with enemas) were computed and compared, and their corresponding DVH parameters $\left(\mathrm{D}_{0.1 \mathrm{cc}}, \mathrm{D}_{1 \mathrm{cc}}, \mathrm{D}_{2 \mathrm{cc}}, \mathrm{D}_{5 \mathrm{cc}}\right)$ were assessed. T-tests, paired, and unpaired were used to compare continuous variables. Correlation among them was analyzed with Spearman's test. Dose data is reported with the median and interquartile range (iqr) because some did not show a normal distribution, and data was compared with the Mann-Whitney rank-sum test and the Wilcoxon signed rank-test for unpaired and paired data, respectively. The threshold for significance for all study outcomes was $p<0.05$.

\section{Results}

Between December 2013 and May 2016, 20 consecutive patients with cervical cancer were recruited from those referred for definitive chemo-radiotherapy treatment. Accrual was prematurely stopped with 20 patients, due to the observed lack of dosimetric benefit with the rectal enema administration on our parallel study of rectal enemas during VCB [14]. Table 1 shows patients' characteristics. Pelvic EBRT delivered dose (mean \pm SD) was $47.79 \pm 3.08 \mathrm{~Gy}$. The first 10 patients were treated with a metallic Fletcher-style applicator, and the remaining with a CT/MR compatible Utrecht applicator due to a breakage of the former. No significant differences in the rectal length or intrauterine length loading were observed, whichever type of applicator and enemas were used (Table 2). No significant differences in rectal volume or DVH parameters were observed between no enema 
Table 1. Patients' characteristics

Patients $(n)$

\begin{tabular}{lcc}
\hline Stage & $\mathrm{Ib}$ & 1 \\
\cline { 2 - 3 } & $\mathrm{Ila}$ & 1 \\
\cline { 2 - 3 } & $\mathrm{Ilb}$ & 13 \\
\cline { 2 - 3 } & $\mathrm{IIb}$ & 2 \\
\cline { 2 - 3 } & $\mathrm{IVa}$ & 1 \\
\hline Applicator & Fletcher & 10 \\
\cline { 2 - 3 } & Utrecht & 10
\end{tabular}

and enema fractions. Mean rectal volume was $52.64 \pm$ $15.92 \mathrm{~cm}^{3}$ at the first fraction, without enemas, and $53.16 \pm$ $19.28 \mathrm{~cm}^{3}$ at the second fraction, with enemas $(p=0.8673)$. Table 3 shows rectal volumes and rectal dose parameters according to the type of applicator, Fletcher, or Utrecht, at the first fraction, without enemas, and second fraction, with enemas.

No significant intrauterine length loading differences were observed according to the BT fraction with or without enemas (no enema: $5.47 \pm 0.98 \mathrm{~cm}$; enema: $5.41 \pm 0.73 \mathrm{~cm}$; $p=0.5445)$. A statistically significant correlation existed between rectal volume at the first and second BT fractions (without and with enemas) $(r=0.722, p=0.001)$. Figure 1

Table 3. Rectal volume and rectal dose parameters according to the type of applicator and administration of enemas

\begin{tabular}{lccc} 
Factors & Fletcher & Utrecht & $p$-value \\
\hline Mean rectal volume $(c c)$ & & \\
\hline First fraction & $47.68 \pm 18.13$ & $57.61 \pm 12.29$ & 0.169 \\
\hline Second fraction & $48.74 \pm 20.18$ & $57.57 \pm 18.29$ & 0.774 \\
\hline Rectum $D_{0.1 c c}(G y)$ & & & \\
\hline First fraction & $3.41(1.61)$ & $4.44(1.49)$ & 0.096 \\
\hline Second fraction & $3.82(2.03)$ & $3.48(1.93)$ & 1 \\
\hline Rectum $D_{1 c c}(G y)$ & & & \\
\hline First fraction & $2.72(1.09)$ & $3.56(2.19)$ & 0.131 \\
\hline Second fraction & $2.98(1.2)$ & $2.71(1.6)$ & 0.762 \\
\hline Rectum $D_{2 c c}(G y)$ & & & \\
\hline First fraction & $2.46(0.89)$ & $3.07(0.99)$ & 0.059 \\
\hline Second fraction & $2.63(0.93)$ & $2.39(1.37)$ & 0.706 \\
\hline Rectum $D_{5 c c}(G y)$ & & & \\
\hline First fraction & $1.91(0.71)$ & $2.51(0.64)$ & 0.589 \\
\hline Second fraction & $2.13(0.52)$ & $1.99(1.08)$ & 0.706 \\
\hline & & & \\
\hline
\end{tabular}

Data are expressed as mean \pm SD or as median (iar).

$D_{x c c}$-dose to maximum $x$ volume
Table 2. Brachytherapy technical parameters and rectal dose according to applicator type

\begin{tabular}{lccc} 
Factors & Fletcher & Utretch & $p$-value \\
\hline Rectal length (cm) & & & \\
\hline No enema & $8.46 \pm 0.38$ & $8.14 \pm 0.28$ & 0.492 \\
\hline Enema & $8.02 \pm 0.27$ & $7.71 \pm 0.22$ & 0.388 \\
\hline Intrauterine loading (cm) & & \\
\hline No enema & $5.72 \pm 0.26$ & $5.22 \pm 0.35$ & 0.265 \\
\hline Enema & $5.5 \pm 0.19$ & $5.33 \pm 0.27$ & 0.615 \\
\hline$\Delta$ rectal volume (cc) & $-1.06 \pm 3.83$ & $0.04 \pm 4.86$ & 0.862 \\
\hline$\Delta \mathrm{D}_{0.1 \mathrm{cc}}$ (Gy) & $-0.33(1.68)$ & $0.74(2.11)$ & 0.082 \\
\hline$\Delta \mathrm{D}_{1 \mathrm{ccc}}$ (Gy) & $-0.2(0.78)$ & $0.63(1.9)$ & 0.151 \\
\hline$\Delta \mathrm{D}_{2 \mathrm{cc}}$ (Gy) & $-0.07(0.52)$ & $0.52(1.55)$ & 0.151 \\
\hline$\Delta \mathrm{D}_{5 \mathrm{cc}}$ (Gy) & $-0.09(0.55)$ & $0.43(1.14)$ & 0.131
\end{tabular}

Data are expressed as mean $\pm S D$ or as median (iqr), $\Delta$-difference, $D_{x c c}-$ dose to maximum $x$ volume

$\Delta D_{x c c}: D V H$ difference $-\Delta D V H_{\text {parameter }}=D V H_{\text {No enema }}-D V H_{\text {Enema }}$

shows a regression line between the two volumes. Table 4 shows the rectal volume at the first (without enemas) and second BT fractions (with enemas). The majority of the patients had empty rectums even without enemas and Fleet enemas had a limited effect in cleaning the rectums. All rectal DVH parameters were higher with the rectal enemas, but were not statistically significant (Table 5). No statistically significant correlation was observed between rectal volume change and DVH parameters change due to the BT fraction ( $r$ values, 0.091, 0.123, 0.09, 0.091 for $\mathrm{D}_{0.1 \mathrm{cc}}, \mathrm{D}_{1 \mathrm{cc}}, \mathrm{D}_{2 \mathrm{cc}}$ and $\mathrm{D}_{5 \mathrm{cc}}$, respectively). Figure 2 shows the rectal volume and $\mathrm{D}_{2 \mathrm{cc}}$ according to the BT fraction without enemas and with enemas.

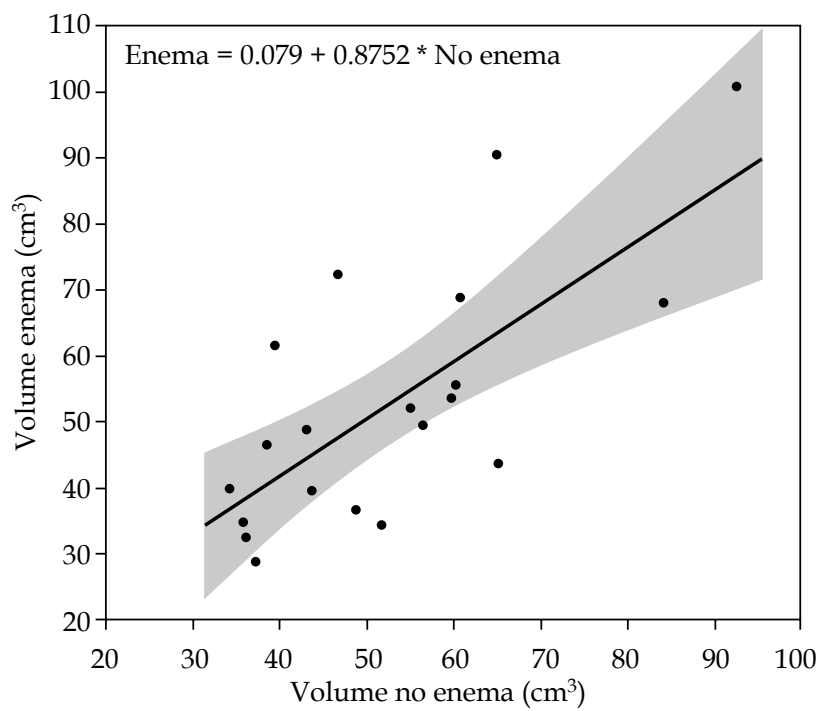

Fig. 1. Scatter plot of the rectal volume at first (without enemas) and second (with enemas) brachytherapy fraction 
Table 4. Rectum content at first brachytherapy fraction without enemas and at second brachytherapy fraction with enemas. The Table shows that the majority of the patients had empty rectums even without enemas as well as the limited emptying effect of the enemas in this population

\begin{tabular}{llccccc} 
& \multicolumn{5}{c}{ First fraction without enema $(n)$} \\
\cline { 2 - 7 } & & Empty & Feces & Air & Air + Feces & Total \\
\hline $\begin{array}{l}\text { Second fraction after } \\
\text { the enema } \\
\text { administration }(n)\end{array}$ & Empty & 9 & 1 & 2 & 0 & 12 \\
\cline { 2 - 7 } & Feces & 1 & 1 & 0 & 0 & 3 \\
\cline { 2 - 7 } & Air & 1 & 1 & 0 & 1 & 3 \\
\cline { 2 - 7 } & Air + Feces & 11 & 5 & 2 & 2 & 20 \\
\cline { 2 - 7 } & Total & & & & 0 & 2 \\
\end{tabular}

\section{Discussion}

In this study, we evaluated the clinical value of rectal enemas administered prior to the brachytherapy procedure in cervical cancer. Our results shows a lack of statistically significant impact of rectal enemas prior to fractionated HDR brachytherapy on DVH parameters.

The ESTRO [9] recommends avoiding rectal discharge if the brachytherapy lasts longer than one or two days. After the introduction of fractionated HDR brachytherapy, a Fleet enema prior to the procedure has remained in clinical practice in several radiotherapy departments. Despite the introduction of the fractionated and faster HDR techniques, some departments have kept on its use in order to avoid bowel movements and rectal anterior wall displacements towards the vagina and cervix. A similar situation occurs with the VCB. Then we started a parallel project with the aim to evaluate the dosimetric value of rectal enemas during modern fractionated therapy in two HDR gynecological brachytherapy settings, the postop-

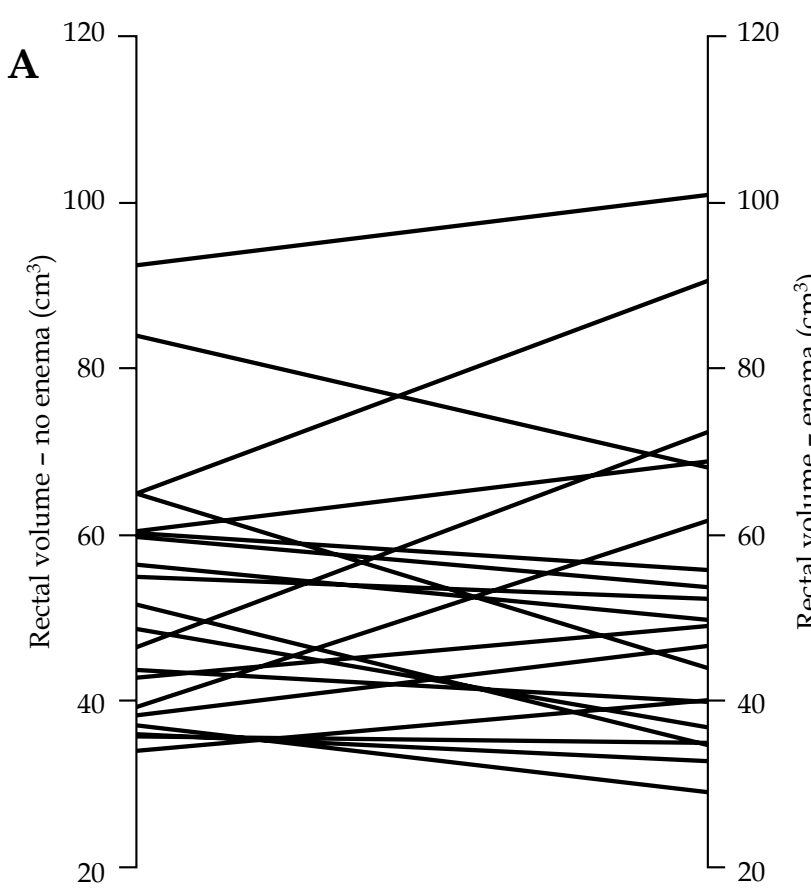

Fig. 2. Spaghetti plot showing rectal volume variation (A) and rectal $D_{2 c c}$ dose variation (B) according to the brachytherapy fraction, without and with Fleet enemas
Table 5. Analyzed parameters at first (without enemas) and second (with enemas) brachytherapy fraction

\begin{tabular}{lccc} 
& No enema & Enema & $p$-value \\
\hline$D_{0.1 c c}(G y)$ & $4.17(2.19)$ & $3.61(1.92)$ & 0.737 \\
\hline$D_{1 c c}(G y)$ & $3.23(1.45)$ & $2.87(1.4)$ & 0.881 \\
\hline$D_{2 c c}(G y)$ & $2.9(1.19)$ & $2.54(1.15)$ & 0.823 \\
\hline$D_{5 c c}(G y)$ & $2.35(0.98)$ & $2.05(0.83)$ & 0.601
\end{tabular}

Data are expressed as median (iqr). $D_{x c c}$-dose to maximum $x$ volume

erative VCB [14] and radical brachytherapy for cervical cancer. Previous studies showing a positive correlation between rectal distension and DVH rectal parameters, either in VCB $[11,13,16]$ or in radical cervical cancer treatment [10] are the background for our project. During VCB it has been demonstrated that gas pocket evacuation reduced rectal volume by a third on average, which lead to

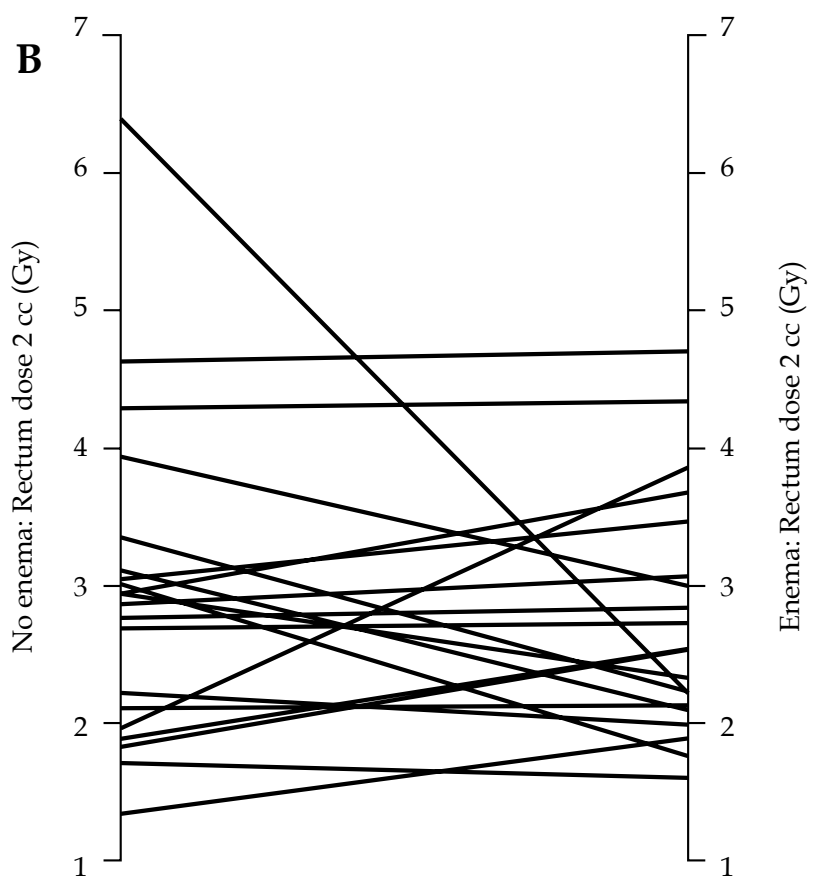


reductions in DVH parameters [13]. In the opposite way, the use of rectal contrast has been linked to a $20 \%$ increase statistically on the rectal volume, which also translated to an increase in all DVH parameters analyzed [11]. A study into cervical cancer at the University of California has shown a significant $\mathrm{D}_{2 \mathrm{cc}}$ increase of $6.58 \mathrm{~Gy}$ for each additional centimeter of distension [10].

Our study was closed prematurely after the analysis of the parallel study on VCB [14] that failed to demonstrate a rectal DVH improvement with the Fleet rectal enema administration despite a significant $15 \%$ reduction in mean rectal volume. The study also suggested that it was ineffective for $35.6 \%$ of patients due to the increase in rectal volume after its administration. The results presented here are consistent with the study carried out in the VCB setting. No rectal DVH differences were observed with the use of Fleet enemas, and some patients also experienced a rec -tal volume increase with Fleet enema administration (Figure 2A). Unlike the previous study, no significant mean rectal volume reduction was seen with Fleet enemas, despite the similar mean rectal volume at the basal status (rectal volume in the VCB study: $52.14 \mathrm{~cm}^{3}$ basal, $44.07 \mathrm{~cm}^{3}$ post-enema). The different percentage of patients treated with EBRT as well as the timing between EBRT and BT could be partially associated to the number of patients with empty rectums. All cervical cancer patients underwent simultaneous EBRT and BT, while nearly half of the endometrial cancer patients only underwent BT. In addition, the use of concomitant chemotherapy for cervical cancer has been linked to a twofold increase in gastrointestinal toxicity [17]. In the prostate EBRT setting, Engels described two patterns of rectal distention during an irradiation course [18]. One of them with large and variable rectal filling. Some could argue against administering rectal enemas if patients had already empty rectums. Nevertheless, it was an unexpected result found during analysis. The former parallel study for endometrial cancer shown that $27 \%$ of patients had an empty rectum in the first fraction without enemas, and $22 \%$ of the patients had an empty rectum in both fractions [14]. Although the lack of benefit in the overall group, Figure 2 suggest that some individual patients could profit from this harmless procedure.

The premature ending of the study is the main limitation of our work, as it reduces statistical power. The main reason to stop the study was that $35.6 \%$ of the endometrial cancer patients showed an increase in rectal volume after enema administration. The experimental design of the study, with paired data belonging to the same patient analyzing the first and second BT fractions, meant that every patient was submitted to the basal and experimental conditions, precluding any toxicity analysis. This study design impeded to discover the extent of the empty rectums in an early-stage. In order to discover it, a basal CT followed by the rectal enema and a second CT set would be needed. A strong correlation between rectal dose and rectal toxicity has been demonstrated $[19,20,21,22]$, so dose accumulation procedures were created to compute accurate total doses to OAR after gynecological brachytherapy $[23,24]$. Nevertheless, the similar doses obtained by both experimental conditions suggest that differences in rectal toxicity would be hard to demonstrate. Other factors that can modify DVH doses, such as the applicator position $[10,25]$, the body mass index $[26,27]$, or the bladder volume [28] were out of the scope of the study. These factors have not been extensively studied and results are contradictory among the few published reports. Although only CT was used for image segmentation, the use of MRI has proven to modify tumor volumes and absorbed doses [29], therefore MRI with the applicator in place is the gold standard in real image for brachytherapy. Many institutions cannot carry this out in clinical routines, therefore other strategies have been developed, such as the use of CT/MR images on alternate applications [30] or the use of MRI acquired outside a radiotherapy protocol [31]. We do not believe that the use of MRI would modify rectum doses, because recently published results failed to demonstrate rectal and bladder dose differences related to the segmentation with CT or MRI [32,33]. Future work needs to address the effect of rectal volume in a large population of patients treated with brachytherapy for locally advanced cervical cancer, and evaluate how it effects total dose accumulation.

\section{Conclusions}

Our results suggest that while a rectal evacuation might be useful during long-lasting bed rest low-doserate brachytherapy, the quicker HDR techniques carried out in an outpatient setting have no need for it. These results are in accordance with the parallel trial on VCB. Our rectal cleansing enemas protocol was unable to find any dosimetric improvement. Perhaps administering rectal enemas immediately before each brachytherapy application might bring a reduction in rectal doses. We believe that more studies in this field are necessary because of the disagreement with some of our previous retrospective studies. Nevertheless, reasons for the use of rectal enemas remain like hygiene, when interstitial implants are combined to the intracavitary procedure.

\section{Acknowledgement}

We are grateful to Phil Hoddy for language assistance.

\section{Disclosure}

Authors report no conflict of interest.

\section{References}

1. Wang $X$, Li J, Wang $P$ et al. Image guided radiation therapy boost in combination with high-dose-rate intracavitary brachytherapy for the treatment of cervical cancer. J Contemp Brachytherapy 2016; 8: 122-127.

2. Okuma K, Yamashita H, Kobayashi R et al. A study of highdose-rate intracavitary brachytherapy boost for curative treatment of uterine cervical cancer. J Contemp Brachytherapy 2015; 7: 128-134.

3. Teke F, Yoney A, Teke M et al. Evaluation of outcome and prognostic factors in 739 patients with uterine cervix carcinoma: a single institution experience. Contemp Oncol (Pozn) 2015; 19: 130-136.

4. Tan PW, Koh VY, Tang JI. Outpatient combined intracavitary and interstitial cervical brachytherapy: barriers and solutions 
to implementation of a successful programme - a single institutional experience. J Contemp Brachytherapy 2015; 7: 259-263.

5. Maduro JH, Pras E, Willemse PH et al. Acute and long-term toxicity following radiotherapy alone or in combination with chemotherapy for locally advanced cervical cancer. Cancer Treat Rev 2003; 29: 471-488.

6. Hyun Kim T, Choi J, Park SY et al. Dosimetric parameters that predict late rectal complications after curative radiotherapy in patients with uterine cervical carcinoma. Cancer 2005; 104: 1304-1311.

7. Kim RY, Shen S, Duan J. Image-based three-dimensional treatment planning of intracavitary brachytherapy for cancer of the cervix: dose-volume histograms of the bladder, rectum, sigmoid colon, and small bowel. Brachytherapy 2007; 6: 187-194.

8. Cheng JC-H, Peng L-C, Chen Y-H et al. Unique role of proximal rectal dose in late rectal complications for patients with cervical cancer undergoing high-dose-rate intracavitary brachytherapy. Int J Radiat Oncol Biol Phys 2003; 57: 1010-1018.

9. Gerbaulet A, Potter R, Mazeron JJ et al. The GEC ESTRO Handbook of Brachytherapy. ESTRO, Brussels 2002; 301-363.

10. Lim J, Durbin-Johnson B, Valicenti R et al. The impact of maximum rectal distention and tandem angle on rectal dose delivered in 3D planned gynecologic high dose-rate brachytherapy. Int J Gynecol Cancer 2013; 23: 1078-1083.

11. Sabater S, Arenas M, Berenguer R et al. Dosimetric analysis of rectal filling on rectal doses during vaginal cuff brachytherapy. Brachytherapy 2015; 14: 458-463.

12. Sabater S, Andres I, Jimenez-Jimenez E et al. Rectal contrast increases rectal dose during vaginal cuff brachytherapy. Brachytherapy 2016; 15: 35-39.

13. Sabater S, Sevillano MM, Andres I et al. Reduction of rectal doses by removal of gas in the rectum during vaginal cuff brachytherapy. Strahlenther Onkol 2013; 189: 951-956.

14. Sabater S, Andres I, Gascon M et al. Effect of rectal enemas on rectal dosimetric parameters during high-dose-rate vaginal cuff brachytherapy: A prospective trial. Strahlenther Onkol 2016; 192: 248-253.

15. Nag S, Erickson B, Thomadsen B et al. The American Brachytherapy Society recommendations for high-dose-rate brachytherapy for carcinoma of the cervix. Int J Radiat Oncol Biol Phys 2000; 48: 201-211.

16. Sabater S, Andres A, de la Vara Olivas V et al. Effect of rectal distention on vaginal cuff brachytherapy. Radiother Oncol 2013; 106: s365-366.

17. Kirwan JM, Symonds P, Green JA et al. A systematic review of acute and late toxicity of concomitant chemoradiation for cervical cancer. Radiother Oncol 2003; 68: 217-226.

18. Engels B, Tournel K, Soete G et al. Assessment of rectal distention in radiotherapy of prostate cancer using daily megavoltage CT image guidance. Radiother Oncol 2009; 90: 377-381.

19. Chen SW, Liang JA, Yeh LS et al. Comparative study of reference points by dosimetric analyses for late complications after uniform external radiotherapy and high-dose-rate brachytherapy for cervical cancer. Int J Radiat Oncol Biol Phys 2004; 60: 663-671.

20. Georg P, Kirisits C, Goldner G et al. Correlation of dose-volume parameters, endoscopic and clinical rectal side effects in cervix cancer patients treated with definitive radiotherapy including MRI-based brachytherapy. Radiother Oncol 2009; 91: 173-180.

21. Rovirosa A, Ascaso C, Arenas M et al. Can we shorten the overall treatment time in postoperative brachytherapy of endometrial carcinoma? Comparison of two brachytherapy schedules. Radiother Oncol 2015; 116: 143-148.

22. Rios I, Rovirosa A, Ascaso C et al. Vaginal-cuff control and toxicity results of a daily HDR brachytherapy schedule in endometrial cancer patients. Clin Transl Oncol 2016; 18: 925-930.
23. Sabater S, Andres I, Sevillano M et al. Dose accumulation during vaginal cuff brachytherapy based on rigid/deformable registration vs. single plan addition. Brachytherapy 2014; 13: 343-351.

24. Andersen ES, Noe KØ, Sørensen TS et al. Simple DVH parameter addition as compared to deformable registration for bladder dose accumulation in cervix cancer brachytherapy. Radiother Oncol 2013; 107: 52-57.

25. Hoskin PJ, Cook M, Bouscale D et al. Changes in applicator position with fractionated high dose rate gynaecological brachytherapy. Radiother Oncol 1996; 40: 59-62.

26. Sabater S, Arenas M, Berenguer R et al. Body Mass Index and Doses at Organs at Risk in a Mediterranean Population Treated with Postoperative Vaginal Cuff Brachytherapy. Cancer Res Treat 2015; 47: 473-479.

27. Lim J, Durbin-Johnson B, Valicenti R et al. The impact of body mass index on rectal dose in locally advanced cervical cancer treated with high-dose-rate brachytherapy. Brachytherapy 2013; 12: 550-554.

28. Siavashpour Z, Aghamiri MR, Jaberi R et al. Optimum organ volume ranges for organs at risk dose in cervical cancer intracavitary brachytherapy. J Contemp Brachytherapy 2016; 8: 135-142.

29. Viswanathan AN, Dimopoulos J, Kirisits C et al. Computed tomography versus magnetic resonance imaging-based contouring in cervical cancer brachytherapy: results of a prospective trial and preliminary guidelines for standardized contours. Int J Radiat Oncol Biol Phys 2007; 68: 491-498.

30. Dolezel M, Odrazka K, Zizka J et al. MRI-based preplanning using CT and MRI data fusion in patients with cervical cancer treated with 3D-based brachytherapy: feasibility and accuracy study. Int J Radiat Oncol Biol Phys 2012; 84: 146-152.

31. Sabater S, Pastor-Juan Mdel R, Berenguer R et al. Analysing the integration of MR images acquired in a non-radiotherapy treatment position into the radiotherapy workflow using deformable and rigid registration. Radiother Oncol 2016; 119: 179-184.

32. Krishnatry R, Patel FD, Singh P et al. CT or MRI for image-based brachytherapy in cervical cancer. Jpn J Clin Oncol 2012; 42: 309-313.

33. Eskander RN, Scanderbeg D, Saenz CC et al. Comparison of computed tomography and magnetic resonance imaging in cervical cancer brachytherapy target and normal tissue contouring. Int J Gynecol Cancer 2010; 20: 47-53. 